\title{
Status of chiral perturbation theory for light mesons
}

\author{
Gerhard Ecker \\ University of Vienna, Faculty of Physics, Boltzmanngasse 5, A-1090 Wien, Austria \\ E-mail: gerhard.ecker@univie.ac.at
}

\begin{abstract}
The status of chiral perturbation theory in the meson sector is illustrated with several topical examples. The longtime discrepancy between theory and experiment for the charged pion polarizabilities has now been resolved in favour of the chiral $S U(2)$ result to next-to-next-to-leading order. For chiral $S U(3)$, the main obstacles are the large number of badly known coupling constants (LECs) and the lack of convergence of the low-energy expansion in many cases of interest. I describe a new global fit of the LECs in the strong sector that leads to a prediction of the CKM matrix element $V_{u s}$ in agreement with the latest lattice determinations. The slow convergence of the chiral series is particularly acute in transitions with strong final-state interactions calling for dispersive treatments. The status of dispersive approaches is reviewed for $K_{\ell 4}$ decays and for $\eta \rightarrow 3 \pi$ decays where precise measurements of Dalitz plot distributions have recently become available.
\end{abstract}

The 8th International Workshop on Chiral Dynamics, CD2015

29 June 2015 - 03 July 2015

Pisa, Italy 


\section{Introduction}

Chiral perturbation theory (CHPT) in its original form $[1,2,3]$ describes the strong, electromagnetic (external photons) and semileptonic weak interactions at low energies for the light pseudoscalar mesons, pions only for chiral $S U(2)$, the light pseudoscalar octet for chiral $S U(3)$. Later on, the CHPT scheme was extended to account also for the nonleptonic weak interactions and for radiative corrections, requiring the incorporation of dynamical photons and leptons. A brief review of the relevant chiral Lagrangians can be found in Ref. [4]. Schematically, they are displayed in Table 1.

\begin{tabular}{|c|c|}
\hline $\mathscr{L}_{\text {chiral order }}$ \# of LECs $)$ & loop order \\
\hline $\mathscr{L}_{p^{2}}(2)+\mathscr{L}_{p^{4}}^{\text {odd }}(0)+\mathscr{L}_{G_{F} p^{2}}^{\Delta S=1}(2)+\mathscr{L}_{G_{8} e^{2} p^{0}}^{\text {emeak }}(1)$ & $L=0$ \\
$+\mathscr{L}_{e^{2} p^{0}}^{\text {em }}(1)+\mathscr{L}_{\text {kin }}^{\text {leptons }}(0)$ & \\
$+\mathscr{L}_{p^{4}}(10)+\mathscr{L}_{p^{6}}^{\text {odd }}(23)+\mathscr{L}_{G_{8} p^{4}}^{\Delta S S=1}(22)+\mathscr{L}_{G_{27} p^{4}}^{\Delta S=1}(28)$ & $L \leq 1$ \\
$+\mathscr{L}_{G_{8} e^{2} p^{2}}^{\text {emweak }}(14)+\mathscr{L}_{e^{2} p^{2}}^{\text {em }}(13)+\mathscr{L}_{e^{2} p^{2}}^{\text {leptons }}(5)$ & \\
$+\mathscr{L}_{p^{6}}(90)$ & $L \leq 2$ \\
\hline
\end{tabular}

Table 1: Effective chiral Lagrangian in the meson sector for chiral $S U(3)$, with the number of LECs in brackets.

Most of the strong, electromagnetic or semileptonic weak processes have been calculated up to next-to-next-to-leading order (NNLO), which includes one- and two-loop contributions. For a review of CHPT at the two-loop level see Ref. [5], for a repository of corresponding amplitudes, which is regularly being updated, see Ref. [6]. For the nonleptonic weak interactions and for radiative corrections, complete calculations exist in general only at NLO (one-loop level) although attempts at going beyond have been made in several cases (unitarity corrections, dispersion theory, etc.).

Except for the technical complications of higher orders, the main obstacle is the rapidly growing number of coupling constants usually denoted as low-energy constants (LECs), characteristic for an effective (nonrenormalizable) quantum field theory. As shown in Table 1, only the strong chiral Lagrangian has been carried to the NNLO level precisely for that reason. For instance, for the nonleptonic weak Lagrangian even many NLO LECs are poorly known. In the strong sector, some progress has recently been made to estimate many LECs up to NNLO. This will be reviewed in Sec. 2.

In addition to badly known LECs, the lack of "convergence" of the chiral expansion in many cases is the second major obstacle on the way to reliable predictions. This refers mostly to calculations in chiral $S U$ (3) where the natural expansion parameter is $M_{K}^{2} /\left(4 \pi F_{\pi}\right)^{2} \simeq 0.20$. In fact, there are several examples where successive orders in chiral $S U(3)$ increase by substantially more than $20 \%$. Prominent examples are $\eta \rightarrow 3 \pi$ decays that will be discussed at length in Sec. 6. Instead of calculating still higher orders in CHPT, the emphasis in the field has shifted towards dispersive 
approaches, especially in cases with strong rescattering in the final state. This status report bears ample evidence for the growing importance of dispersive treatments in combination with CHPT.

Although the activity in the field has decreased during the past 10 years both in theory and experiment, there are several key experiments still running with a large impact on the field. Two of the most important recent experimental advances in our field were presented at this Workshop and will be covered in this talk: the COMPASS experiment measuring the charged pion polarizabilities and several recent experiments investigating $\eta \rightarrow 3 \pi$ decays.

Here is an outline of the talk. In Sec. 2 I will discuss the status of LECs in the strong sector. I will describe our recent attempts to get a better handle on the LECs of both NLO and NNLO by means of a global fit yielding a preferred set of LECs termed BE14. The status of CKM unitarity is reviewed in Sec. 3. The longstanding problem of the discrepancy between theory and experiment for the charged pion polarizabilities has been resolved recently by the results of the COMPASS experiment. The history of this problem is briefly recalled in Sec. 4. Moving up in meson masses, recent activities in the semileptonic $K_{\ell 4}$ decays are reviewed in Sec. 5. To properly describe $\eta \rightarrow 3 \pi$ decays is still a major problem for CHPT, especially in view of recent precise data for the Dalitz plot distributions. Various dispersive approaches trying to improve on the NNLO CHPT amplitudes are discussed in Sec. 6.

\section{Low-energy constants}

\begin{tabular}{|c|r|r|}
\hline & $C_{i}^{r}=0$ & Ref. [10] \\
\hline $10^{3} L_{1}^{r}$ & $0.67(06)$ & $0.45(07)$ \\
$10^{3} L_{2}^{r}$ & $0.17(04)$ & $0.22(04)$ \\
$10^{3} L_{3}^{r}$ & $-1.76(21)$ & $-1.66(22)$ \\
$10^{3} L_{4}^{r}$ & $0.73(10)$ & $0.51(12)$ \\
$10^{3} L_{5}^{r}$ & $0.65(05)$ & $2.61(12)$ \\
$10^{3} L_{6}^{r}$ & $0.25(09)$ & $0.73(06)$ \\
$10^{3} L_{7}^{r}$ & $-0.17(06)$ & $-0.54(05)$ \\
$10^{3} L_{8}^{r}$ & $0.22(08)$ & $1.43(10)$ \\
\hline$\chi^{2}$ & 26 & 25 \\
dof & 9 & 9 \\
\hline
\end{tabular}

Table 2: Fits for NLO LECs $L_{i}^{r}(\mu)$ for two special cases with fixed NNLO LECs $C_{i}^{r}(\mu)$. In the second column, all $C_{i}^{r}$ are set to zero. In the third column, we use the $C_{i}^{r}$ obtained from a chiral quark model [10], itself an update of a previous attempt [11] to determine the $C_{i}^{r}$. The renormalization scale is always $\mu=0.77 \mathrm{GeV}$.

Chiral perturbation theory as the prototype of an effective field theory depends on a large number of coupling constants, increasing rapidly at higher orders. The relevant chiral Lagrangians in the meson sector, with the associated number of LECs in brackets, are listed in Table 1 for chiral $S U(3)$.

A comprehensive survey of mesonic LECs can be found in Ref. [4]. In this talk, I will concentrate on a new determination of NLO and NNLO LECs in the strong sector [4] marked in red in Table 1. This new determination is both an update and an extension of the previous global fit by 
Bijnens and Jemos [7]. Referring to Ref. [7] for the general strategy of the fit, I discuss here only the new ingredients of our approach [4].

- In addition to the 13 observables employed by Bijnens and Jemos, we have also used the relations between the chiral $S U(2) \operatorname{LECs} l_{j}(j=1, \ldots, 4)$ [2] and the $S U(3)$ LECs $L_{i}$ [3] and $C_{i}$ [8] obtained in Ref. [9].

- The altogether 17 input data depend on most of the $L_{i}(i=1, \ldots, 8)$ and on 34 combinations of the $C_{i}$. It is therefore obvious that one has to make some assumptions about the $C_{i}$ in order to proceed. That this is a nontrivial task becomes evident from Table 2 where we consider two special cases with fixed values for the $C_{i}$.

Both choices for the $C_{i}^{r}$ in Table 2 clearly lead to unacceptable fits. In addition to the large values of $\chi^{2}$, the LECs $L_{4}^{r}, L_{6}^{r}$ and $2 L_{1}^{r}-L_{2}^{r}$ show no sign of large- $N_{c}$ suppression.

In order to proceed, we had to make some assumptions about the NNLO LECs. For most of the 34 combinations of the $C_{i}$ appearing in our 17 input variables, theoretical predictions exist in the literature (see Ref. [4]), although in some cases conflicting with each other. We have used the available information to define priors for the $C_{i}$ with associated ranges of acceptable values. We then use an iterative procedure employing two different methods (minimization or random walk) in the restricted space of the $C_{i}$, with essentially the same results: if the fitted values of the $L_{i}$ deviate too much from the NLO fit results (shown in the fourth column of Table 3 ) and/or if the $\chi^{2}$ is too large, we modify the boundaries of the $C_{i}$ space and start again. To speed up the convergence of this procedure, we introduced a penalty function for bad convergence of the meson masses. On the other hand, we did not enforce large $N_{c}$ on the $C_{i}$ from the outset.

\begin{tabular}{|r||r|r||r|r|}
\hline & NNLO free fit & NNLO BE14 & NLO 2014 & GL 1985 [3] \\
\hline $10^{3} L_{A}^{r}$ & $0.68(11)$ & $0.24(11)$ & $0.4(2)$ & \\
$10^{3} L_{1}^{r}$ & $0.64(06)$ & $0.53(06)$ & $1.0(1)$ & $0.7(3)$ \\
$10^{3} L_{2}^{r}$ & $0.59(04)$ & $0.81(04)$ & $1.6(2)$ & $1.3(7)$ \\
$10^{3} L_{3}^{r}$ & $-2.80(20)$ & $-3.07(20)$ & $-3.8(3)$ & $-4.4(2.5)$ \\
$10^{3} L_{4}^{r}$ & $0.76(18)$ & 0.3 & $0.0(3)$ & $-0.3(5)$ \\
$10^{3} L_{5}^{r}$ & $0.50(07)$ & $1.01(06)$ & $1.2(1)$ & $1.4(5)$ \\
$10^{3} L_{6}^{r}$ & $0.49(25)$ & $0.14(05)$ & $0.0(4)$ & $-0.2(3)$ \\
$10^{3} L_{7}^{r}$ & $-0.19(08)$ & $-0.34(09)$ & $-0.3(2)$ & $-0.4(2)$ \\
$10^{3} L_{8}^{r}$ & $0.17(11)$ & $0.47(10)$ & $0.5(2)$ & $0.9(3)$ \\
\hline$F_{0}[\mathrm{MeV}]$ & 64 & 71 & & \\
\hline
\end{tabular}

Table 3: Various fits for the NLO LECs $L_{i}^{r}(i=1, \ldots, 8)$. The values for the large- $N_{c}$ suppressed combination $L_{A}^{r}:=2 L_{1}^{r}-L_{2}^{r}$ are shown separately. We suggest to use fit BE14 [4] in NNLO chiral $S U(3)$ calculations. The last two columns confront an up-to-date NLO fit with the original values from Ref. [3].

At the end of the iteration we perform a standard $\chi^{2}$ fit for the $L_{i}$ with fixed "best" values of the $C_{i}$. The results are shown in Table 3 in the second and third columns. For comparison, we also exhibit an NLO fit (4th column) and the original values of Gasser and Leutwyler [3] (last column). 
Referring to Ref. [4] for more details, I include a few comments here.

- All fits are very sensitive to $L_{4}$ and tend to lead to values incompatible with large $N_{c}$. This tendency is well known from previous global fits and can be understood to some extent from the structure of the chiral Lagrangian [12]. We have therefore enforced small values of $L_{4}$ as supported by lattice studies [13]. It turns out that $L_{6}^{r}$ and $L_{A}^{r}:=2 L_{1}^{r}-L_{2}^{r}$ are then automatically suppressed as well.

- The quality of both NNLO fits in Table 3 is excellent. They only make sense with associated sets of the $C_{i}$ (see Ref. [4]).

- Once a decent convergence is enforced for the meson masses, we find reasonable convergence for the other observables. The fitted $L_{i}$ together with the "best" values of the $C_{i}$ show again qualitative evidence for resonance saturation [14]. In particular, $\eta^{\prime}$ dominance is observed for some of the $C_{i}$ in accordance with large $N_{c}$ [15].

- Our preferred fit BE14 is remarkably consistent with the NLO fits in the last two columns of Table 3 spanning a period of nearly 30 years.

For a determination of other LECs (not covered by our fits) from $\tau$ decay data I refer to the contribution of Golterman [16].

\section{Status of CKM unitarity}

Violation of unitarity of the three-generation Cabibbo-Kobayashi-Maskawa mixing matrix would be a clear indication for physics beyond the Standard Model. The most sensitive test involves the first row of the CKM matrix with elements $V_{u d}, V_{u s}$ and $V_{u b}$. In view of the present sensitivity, only $V_{u d}$ and $V_{u s}$ are relevant for the unitarity test.

Table 4 collects the different sources of input for $V_{u d}$ and $V_{u s}$.

\begin{tabular}{|c|c|c|}
\hline matrix element & input source & significance \\
\hline$V_{u d}$ & superallowed $\beta$ decays & \\
& neutron $\beta$ decay & \\
& pion $\beta$ decay & \\
& $K_{\ell 3}$ decays $\rightarrow\left|f_{+}^{K^{0} \pi^{-}}(0) V_{u s}\right|$ & \\
$\Gamma\left(K_{\mu 2}\right) / \Gamma\left(\pi_{\mu 2}\right), F_{K} / F_{\pi}, V_{u d}$ & \\
& $\Gamma\left(\tau \rightarrow X_{s} v\right)$ vs. $\Gamma\left(\tau \rightarrow X_{\text {nonstrange }} v\right), V_{u d}$ & \\
& hyperon decays & \\
\hline
\end{tabular}

Table 4: Sources of input for the determination of the CKM matrix elements $V_{u d}$ and $V_{u s}$.

To pin down $V_{u s}$ independently of $V_{u d}$, one needs $f_{+}^{K^{0} \pi^{-}}(0)$, the $K_{\ell 3}$ vector form factor at $t=0$. Some steps in the 30-year-history of estimates/determinations of $f_{+}^{K^{0} \pi^{-}}(0)$ are listed in Fig. 1. 


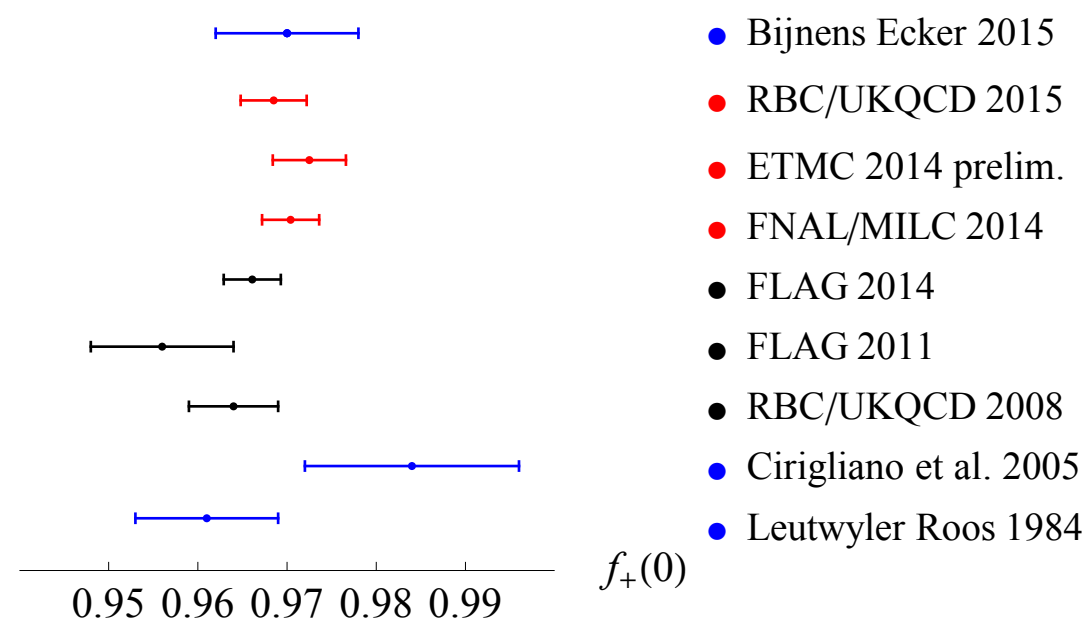

Figure 1: Incomplete list of determinations of $f_{+}^{K^{0} \pi^{-}}(0)$ : Leutwyler Roos 1984 [17], Cirigliano et al. 2005 [18], RBC/UKQCD 2008 [19], FLAG 2011 [20], FLAG 2014 [13], FNAL/MILC 2014 [21], ETMC (preliminary SU(3) analysis) [22], RBC/UKQCD 2015 [23], Bijnens Ecker 2015 [24].

The three most recent lattice determinations are marked in red and will be used for the unitarity test below. Combining the NNLO CHPT calculation of $K_{\ell 3}$ form factors [25] and fit BE14 for the LECs [4], one finds [24]

$$
f_{+}^{K^{0} \pi^{-}}(0)=1-\underbrace{0.02276}_{\text {NLO }}-\underbrace{0.00754}_{\text {NNLO }}
$$

leading to the CHPT prediction

$$
f_{+}^{K^{0} \pi^{-}}(0)=0.970 \pm 0.008
$$

with a very cautious error estimate. This value should be compared with the average of the three most recent lattice determinations (marked in red in Fig. 1)

$$
f_{+}^{K^{0} \pi^{-}}(0)=0.9703 \pm 0.0021
$$

to be used for the following unitarity test $\left(K_{\ell 3} 2015\right.$ in Fig. 2). The other input for Fig. 2 is taken from the contributions of Lusiani [26] and Moulson [27] at CKM2014, except for the most recent update of $V_{u d}$ [28].

Fig. 2 indicates that, instead of worrying about CKM unitarity, one should first try to straighten out the seemingly disparate entries for $V_{u s}$. Especially the extraction from $\tau$ data looks problematic compared to the two precise determinations from $K$ decays. At least among many theorists, the measured branching ratio $B\left(\tau \rightarrow X_{s} v\right)$ is often suspected to be the culprit. Antonelli et al. therefore proposed [29] to use $K$ decays and lepton universality to calculate the decay widths $\Gamma(\tau \rightarrow K v)$ and $\Gamma(\tau \rightarrow K \pi \nu)$, which together make up $68 \%$ of the total strange width. This indeed shifts the $V_{u s}$ entry from $\tau$ decays in the right direction as shown in Fig. 3. For the sake of the argument, I have in addition increased in Fig. 3 the value of $V_{u d}$ from Ref. [28] by $3 \sigma$, anticipating the possible status of CKM unitarity at the time of Chiral Dynamics 2018 .... 


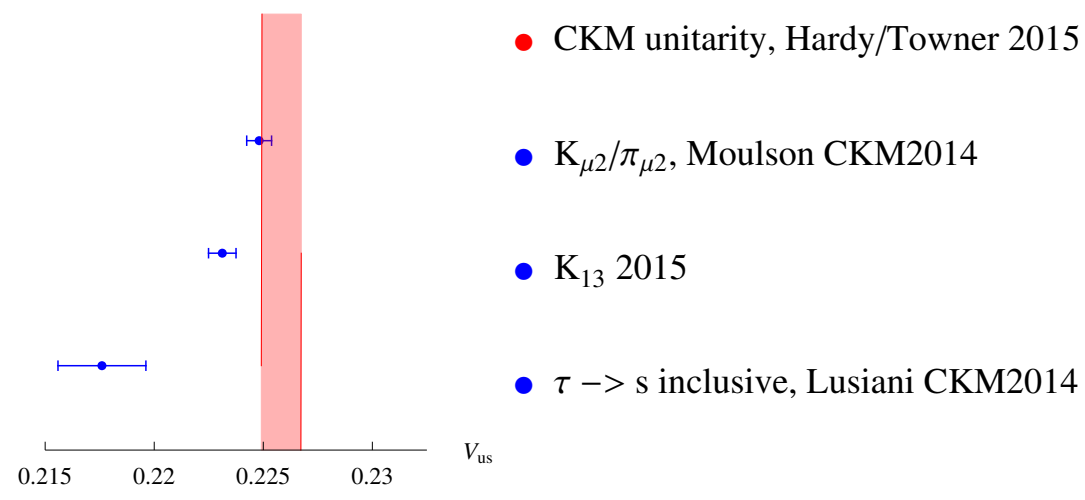

Figure 2: Values for $V_{u s}$ using the various inputs discussed in the text. The red bar indicates the prediction from unitarity with $V_{u d}$ taken from Ref. [28].

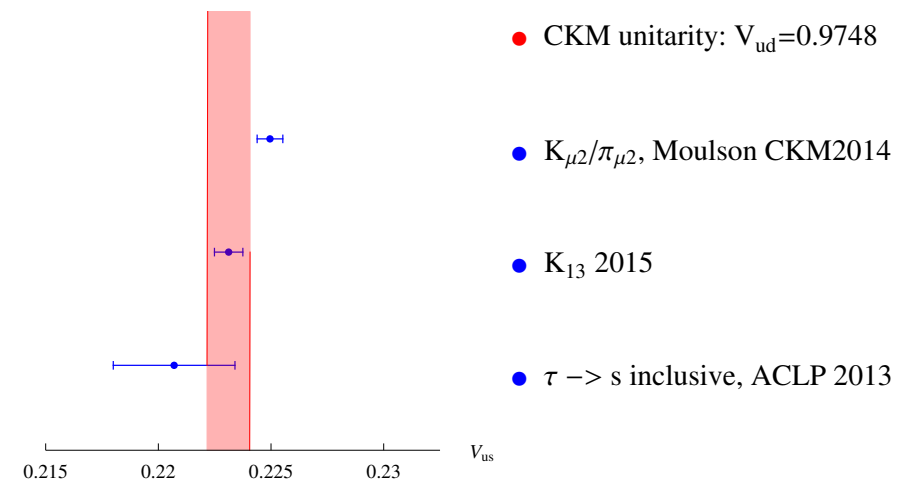

Figure 3: Compared to the previous figure, the value for $V_{u s}$ from $\tau$ decays is now from Antonelli et al. (ACLP 2013) [29] and $V_{u d}=0.97480(21)$ is bigger than the standard value [28] by $3 \sigma$.

\section{Charged pion polarizabilities}

The $\gamma \gamma \pi^{+} \pi^{-}$complex is an early example of NNLO CHPT. Since we are dealing here with chiral $S U(2)$, one expects good convergence: from scalar electrodynamics at lowest $O\left(p^{2}\right)$ to NLO [30] and NNLO [31, 32]. This is indeed borne out by the calculations as exemplified by the total cross section $\sigma\left(\gamma \gamma \rightarrow \pi^{+} \pi^{-}\right)$shown in Fig. 4.

The electric $\left(\alpha_{\pi}\right)$ and magnetic $\left(\beta_{\pi}\right)$ polarizabilities can be defined via the threshold expansion for the differential cross section for pion Compton scattering $\gamma \pi^{ \pm} \rightarrow \gamma \pi^{ \pm}$:

$$
\frac{d \sigma}{d \Omega}=\left(\frac{d \sigma}{d \Omega}\right)_{\text {Born }}-\frac{\alpha M_{\pi}^{3}\left(s-M_{\pi}^{2}\right)^{2}}{4 s^{2}\left(s z_{+}+M_{\pi}^{2} z_{-}\right)}\left(z_{-}^{2}\left(\alpha_{\pi}-\beta_{\pi}\right)+\frac{s}{M_{\pi}^{4}} z_{+}^{2}\left(\alpha_{\pi}+\beta_{\pi}\right)\right)+\ldots
$$

with $s=\left(p_{\pi^{+}}+p_{\pi^{-}}\right)^{2}, z_{ \pm}=1 \pm \cos \theta_{\mathrm{cm}}$.

At NLO in CHPT, electric and magnetic polarizabilities are equal. In addition to the loop contribution, a single combination of $S U(2)$ LECs $2 l_{5}-l_{6}$ enters, which is accurately known from $\pi \rightarrow e v \gamma$ [2]. At NNLO the LECs $l_{1}, l_{2}, l_{3}, l_{4}$ (in one-loop diagrams) and three NNLO LECs contribute together with one-and two-loop contributions. It turns out that the difference $\alpha_{\pi}-\beta_{\pi}$ is not 


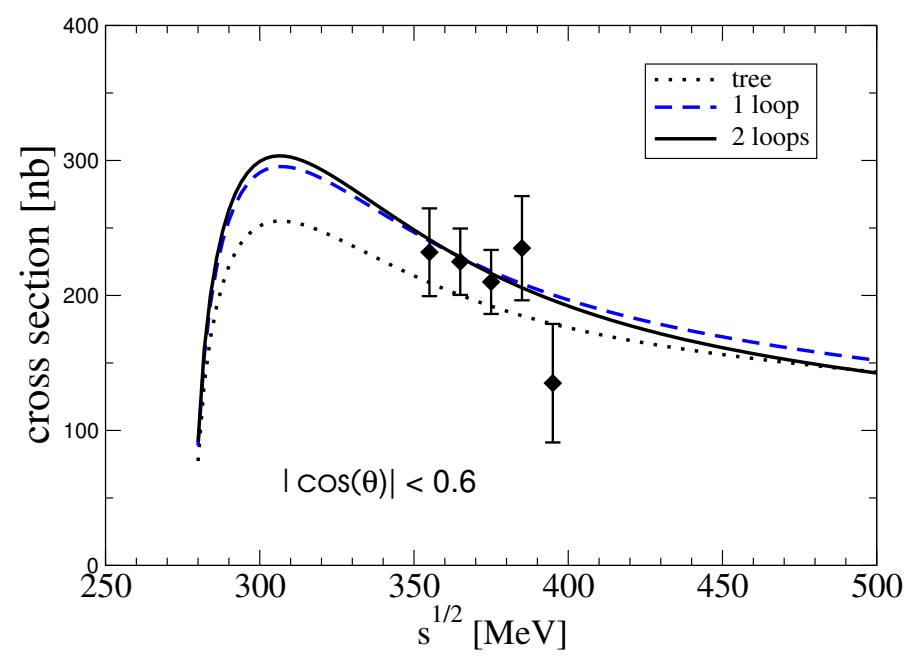

Figure 4: Successive orders of the total cross section $\sigma\left(\gamma \gamma \rightarrow \pi^{+} \pi^{-}\right)$taken from Ref. [32].

very sensitive to the NNLO LECs leading to the final result ${ }^{1} \alpha_{\pi}-\beta_{\pi}=5.7 \pm 1.0$ [32]. The sum $\alpha_{\pi}+\beta_{\pi} \simeq 0.16$ is much smaller but the relative uncertainty is bigger than for the difference. Most experiments actually assume $\alpha_{\pi}=-\beta_{\pi}$ in their analyses.

\begin{tabular}{|c||c|c|}
\hline experiment & & $\alpha_{\pi}-\beta_{\pi}$ \\
\hline MAMI A2 2005 [34] & $\gamma p \rightarrow \gamma \pi^{+} n$ & $11.6 \pm 1.5_{\text {stat }} \pm 3.0_{\text {syst }} \pm 0.5_{\text {mod }}$ \\
COMPASS 2015 $\left(\beta_{\pi}=-\alpha_{\pi}\right)[36]$ & $\pi^{-} \mathrm{Ni} \rightarrow \pi^{-} \gamma \mathrm{Ni}$ & $4.0 \pm 1.2_{\text {stat }} \pm 1.4_{\text {syst }}$ \\
\hline theory & & \\
\hline Fil'kov, Kashevarov 2006 [35] & dispersive & $13.0\left({ }_{-1.9}^{+2.6}\right)$ \\
Gasser, Ivanov, Sainio 2006 [32] & CHPT & $5.7 \pm 1.0$ \\
\hline
\end{tabular}

Table 5: Experimental and theoretical results of the past 10 years for the charged pion polarizabilities.

The experimental situation has been inconclusive for a long time, with large uncertainties $\left(\alpha_{\pi}-\beta_{\pi} \sim 4 \div 53\right)$. For more details I refer to the plenary talk of Friedrich [33]. During the last 10 years two more high-precision experiments were performed. The result of the MAMI experiment of 2005 [34] was incompatible with the chiral prediction for $\alpha_{\pi}-\beta_{\pi}$, but seemed to be supported by a dispersive analysis ${ }^{2}$ soon afterwards [35].

The very recent COMPASS experiment at CERN $[36,33]$ has given a new twist to this long story. Their result for $\alpha_{\pi}-\beta_{\pi}$ (assuming $\alpha_{\pi}=-\beta_{\pi}$ ) displayed in Table 5 disagrees with the MAMI result and it is in excellent agreement with the chiral prediction. The chiral practitioner's favourites are marked in red in Table 5, bringing the longtime puzzle of the charged pion polarizabilities to a

\footnotetext{
${ }^{1}$ Polarizabilities are expressed in units of $10^{-4} \mathrm{fm}^{3}$.

${ }^{2}$ The dispersive method of Ref. [35] was criticized by Pasquini et al. [37].
} 
happy ending. It goes without saying that this success story should be confirmed by an independent experiment.

\section{Kaon decays}

\subsection{Semileptonic $K$ decays}

Since the last Chiral Dynamics Workshop several studies on $K_{\ell 4}$ decays have been undertaken within the CHPT framework. In addition to investigating the $K_{\ell 4}$ form factors and extracting the associated LECs of chiral $S U(3)$, one gets access to the $\pi \pi$ threshold region and thus to the $\pi \pi$ scattering lengths.

For the comparison of theoretical predictions with high-precision experimental data, isospinviolating corrections became more and more important. In particular, to determine the $\pi \pi$ scattering lengths from $K_{e 4}$ data [38], it is essential to account for mass-difference corrections in $\pi \pi$ phase shifts [39]. The $S$-wave scattering lengths from $K_{e 4}$ data are then in excellent agreement with the CHPT+Roy equation analysis [40].

A complete treatment of isospin violation must include radiative corrections. Although such corrections are routinely taken into account in the experimental analysis by means of some Monte Carlo program, a state-of-the-art CHPT treatment was missing until recently. Updating and correcting earlier work [41], Stoffer has now provided the missing link [42]. In addition to the complete isospin-violating one-loop corrections for $K_{\ell 4}$, the calculation is done with an arbitrary cut on the photon energy for semi-inclusive $K_{\ell 4 \gamma}$ decays. The complete isospin-violating mass effects for form factors are also included, reproducing in particular the corrections for the phase shifts [39]. The complete matrix element at NLO including all isospin-violating corrections of $O\left(m_{u}-m_{d}, \alpha\right)$ can then be used by future $K_{\ell 4}$ experiments in their Monte Carlo programs.

In two recent papers [43, 44, 45], Bernard, Descotes-Genon and Knecht have extended the analysis of mass-difference effects for the $\pi \pi$ phase shifts beyond the one-loop approximation. Their starting point is the observation that the phase-shift difference measured by NA48/2 [38] can be related to the theoretical expression [40] as

$$
\left[\delta_{S}(s)-\delta_{P}(s)\right]_{\exp }=\delta_{\mathrm{Roy}}^{S-P}\left(s ; a_{0}^{0}, a_{0}^{2}\right)+\delta_{\mathrm{IB}}^{L=1}(s)
$$

where $\delta_{\mathrm{IB}}^{L=1}(s)$ is the one-loop correction [39]. Beyond one loop, $\delta_{\mathrm{IB}}(s)$ will depend on the scattering lengths $a_{0}^{0}, a_{0}^{2}$ in a nontrivial way so that Eq. (5.1) should in general be replaced by

$$
\left[\delta_{S}(s)-\delta_{P}(s)\right]_{\exp }=\delta_{\text {Roy }}^{S-P}\left(s ; a_{0}^{0}, a_{0}^{2}\right)+\delta_{\mathrm{IB}}\left(s ; a_{0}^{0}, a_{0}^{2}\right) .
$$

The dependence of $\delta_{\mathrm{IB}}\left(s ; a_{0}^{0}, a_{0}^{2}\right)$ on the scattering lengths could therefore introduce a bias in the extraction of $a_{0}^{0}, a_{0}^{2}$ from the data. Of course, in the usual chiral expansion this dependence will be hidden in loop contributions and LECs. Therefore, Bernard et al. set up a dispersive representation of $\delta_{\mathrm{IB}}\left(s ; a_{0}^{0}, a_{0}^{2}\right)$ consisting of two parts [43]: a universal part involving only $\pi \pi$ rescattering and a process-dependent part involving the form factors in the coupled channels. Refitting the NA48/2 data for $K^{ \pm} \rightarrow \pi^{+} \pi^{-} e^{ \pm} v_{e}$ [38] with this dispersive representation, the two contributions partially cancel to give rise to isospin-breaking corrections close to the one-loop case as shown in Table 6. 


\begin{tabular}{|c|c|c|}
\hline$a_{0}^{0}$ & $a_{0}^{2}$ & Ref. \\
\hline $0.221 \pm 0.018$ & $-0.0453 \pm 0.0106$ & {$[43]$} \\
$0.2220(128)_{\text {stat }}(50)_{\text {syst }}(37)_{\text {th }}$ & $-0.0432(86)_{\text {stat }}(34)_{\text {syst }}(28)_{\text {th }}$ & {$[38]$} \\
\hline
\end{tabular}

Table 6: $S$-wave scattering lengths extracted from the NA48/2 data for $K^{ \pm} \rightarrow \pi^{+} \pi^{-} e^{ \pm} v_{e}$ [38]. The entries in the second line are based on the dispersive analysis of Ref. [43], those in the third line are from the NA48/2 Collaboration [38] with one-loop isospin-breaking corrections [39] applied.

Very recently, Colangelo, Passemar and Stoffer have extended the NNLO calculation of $K_{\ell 4}$ decay amplitudes [46] by a dispersive treatment including resummation of $\pi \pi$ and $K \pi$ rescattering effects [47], thereby improving the perturbative treatment of $\pi \pi$ final-state interactions. With the usual assumption of two-particle rescattering with $S$ - and $P$-waves only ("reconstruction theorem" [48]), they determine (most of) the subtraction constants by fitting to the data of the high-statistics experiments E865 [49] and NA48/2 [50]. Isospin breaking is taken into account [42]. By matching to CHPT at both the one- and the two-loop level, values for the NLO LECs $L_{1}, L_{2}, L_{3}$ are determined that are compatible with the global fit BE14 [4]. Unlike the CHPT calculation to NNLO, the dispersive treatment can account for the measured curvature of the $S$-wave projection of the form factor $F$ as shown in Fig. 5.

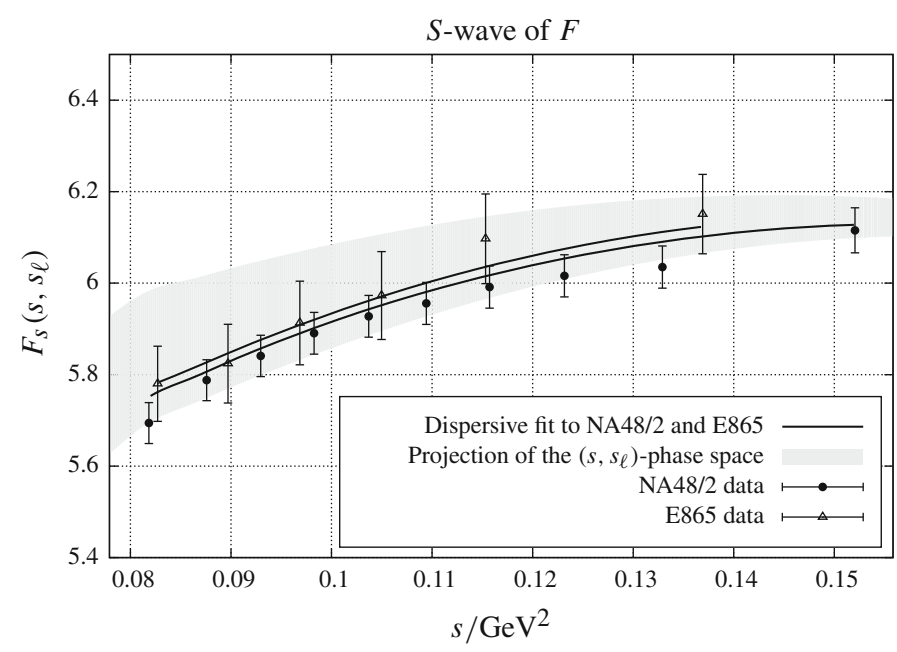

Figure 5: $S$-wave of the $K_{\ell 4}$ form factor $F$. The dispersive description [47] accounts for the measured curvature of the form factor.

\subsection{Nonleptonic $K$ decays}

Since the last Chiral Dynamics Workshop in 2012, there have been no spectacular developments in the CHPT treatment of nonleptonic kaon decays to the best of my knowledge. In this respect, the review of kaon physics in the Standard Model by Cirigliano et al. [51] is therefore still up-to-date. 
Let me nevertheless mention two recent investigations of nonleptonic $K$ decays discussed at this Workshop even though they are not directly related to CHPT. Garron [52] presented the first complete lattice calculation of $K \rightarrow \pi \pi$ with physical kinematics, opening the way for an ab-initio verification of the $\Delta I=1 / 2$ rule and for extracting the parameter $\varepsilon^{\prime}$ measuring direct $\mathrm{CP}$ violation [53]. The lattice value for $\varepsilon^{\prime} / \varepsilon$ comes out substantially smaller than the experimental value [54] but isospin-violating corrections $[55,56]$ still need to be applied.

A completely different, if somewhat unconventional explanation of the $\Delta I=1 / 2$ rule has been proposed by Crewther and Tunstall $[57,58]$ assuming a QCD infrared fixed point.

\section{6. $\eta \rightarrow 3 \pi$ decays}

The decays $\eta \rightarrow \pi^{+} \pi^{-} \pi^{0}, 3 \pi^{0}$ violate isospin. Electromagnetic contributions are known to be small $[59,60]$, but they can be incorporated consistently [61, 62]. To a very good approximation, the decay amplitudes are therefore proportional to the quark mass difference $m_{d}-m_{u}$ :

$$
\begin{gathered}
A(\eta \rightarrow 3 \pi) \sim m_{d}-m_{u} \sim R^{-1} \sim Q^{-2}, \\
R=\frac{m_{s}-m_{u d}}{m_{d}-m_{u}}, Q^{2}=\frac{m_{s}^{2}-m_{u d}^{2}}{m_{d}^{2}-m_{u}^{2}}, \\
Q^{2}=\left(1+\frac{m_{s}}{m_{u d}}\right) R / 2, m_{u d}:=\left(m_{u}+m_{d}\right) / 2 .
\end{gathered}
$$

From the chiral point-of-view, $\eta \rightarrow 3 \pi$ decays are therefore a unique source of information for extracting the light quark mass difference $m_{d}-m_{u}$, little affected by electromagnetic effects. Unfortunately, successive orders in the chiral expansion do not show any sign of convergence as displayed in Table 7.

\begin{tabular}{|c||c|c|c|}
\hline & & $\Gamma\left(\eta \rightarrow \pi^{+} \pi^{-} \pi^{0}\right) / \mathrm{eV}$ & $r$ \\
\hline LO & Osborn, Wallace 1970 [65] & $66^{*}$ & 1.54 \\
NLO & Gasser, Leutwyler 1985 [66] & $160(50)^{*}$ & 1.46 \\
NNLO & Bijnens, Ghorbani 2007 [67] & & 1.47 \\
expt. & PDG 2014 [54] & $300(12)$ & $1.48(5)$ \\
\hline
\end{tabular}

Table 7: Successive orders in the chiral expansion for the decay rate $\Gamma\left(\eta \rightarrow \pi^{+} \pi^{-} \pi^{0}\right)$ and for the ratio $r=$ $\Gamma\left(\eta \rightarrow 3 \pi^{0}\right) / \Gamma\left(\eta \rightarrow \pi^{+} \pi^{-} \pi^{0}\right)$ in comparison with experiment. For the numbers with an asterisk $Q=24.3$ (Dashen's theorem [68]) has been assumed.

In addition, the CHPT amplitudes have problems with the measured Dalitz plot distributions as will be discussed below. It has long been suggested that these problems have to do with the fact that $\pi \pi$ rescattering is included only perturbatively, suggesting a dispersive treatment [63, 64]. Fig. 6 taken from Ref. [64] nicely illustrates the situation for the real part of the decay amplitude: while the chiral corrections from LO to NLO are large, the dispersive effects are actually rather moderate. Fig. 6 also indicates that the decay amplitudes (with or without dispersive corrections) deviate only very little from the Adler zero [69] at $s=u=4 / 3 M_{\pi}^{2}$ as it should be for a chiral $S U$ (2) prediction. 


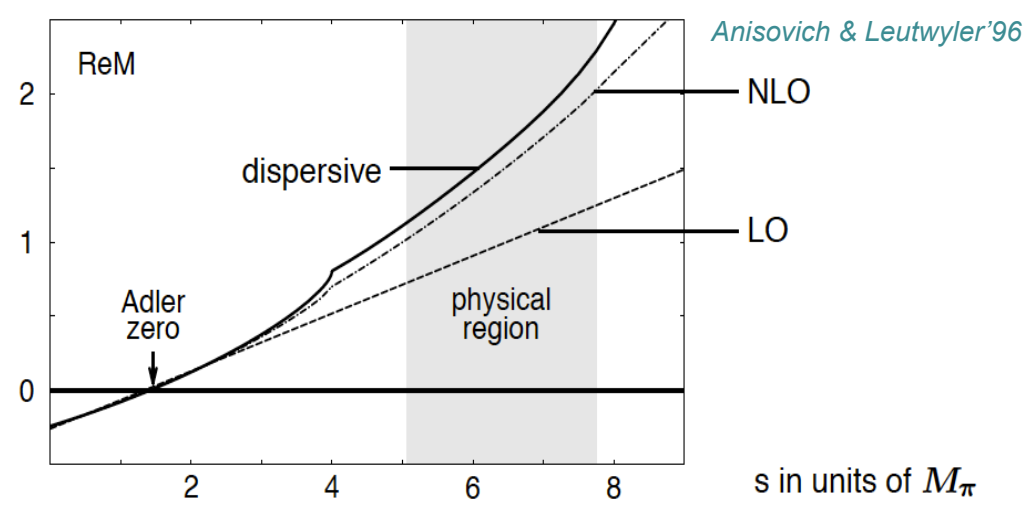

Figure 6: Real part of the (properly normalized) amplitude $M\left(\eta \rightarrow \pi^{+} \pi^{-} \pi^{0}\right)$ along the line $s=u$ : LO (dashed curve), NLO (dash-dotted) and dispersive amplitude (full curve). The figure was taken from Ref. [64].

More recent developments to improve the chiral amplitudes with dispersion theoretic methods are all (with the exception of a recent attempt to include also resonance effects [70]) based on the so-called reconstruction theorem [48], incorporating $\pi \pi$ partial-wave discontinuities for $\ell=0,1$ only. The dispersive amplitudes are then matched to the CHPT amplitudes (mostly at NLO) to obtain the ratios $Q$ or $R$ from the experimental rates. Since the rates cannot be predicted in the dispersive approaches, the crucial tests involve the Dalitz plot parameters defined in Eqs. $(6.2,6.3)$ through an expansion around the center of the Dalitz plot for both charged and neutral modes, especially in view of recent very precise experimental results. In most of the cases to be discussed below, some of the Dalitz plot parameters are also used as input.

The Dalitz plot parameters $a, b, d, \alpha$ are defined via the expansions

$$
\begin{aligned}
\left|A_{\text {charged }}(s, t, u)\right|^{2} & =N\left(1+a Y+b Y^{2}+d X^{2}+\ldots\right), \\
\left|A_{\text {neutral }}(s, t, u)\right|^{2} & =N(1+2 \alpha Z+\ldots)
\end{aligned}
$$

where

$$
\begin{gathered}
X=\frac{\sqrt{3}}{2 M_{\eta} Q_{\eta}}(u-t), Y=\frac{3}{2 M_{\eta} Q_{\eta}}\left(\left(M_{\eta}-M_{\pi^{0}}\right)^{2}-s\right)-1, \\
Z=\left(X^{2}+Y^{2}\right), Q_{\eta}=M_{\eta}-\sum_{i} M_{\pi^{i}} .
\end{gathered}
$$

During the past years, four groups have employed dispersive approaches to analyze $\eta \rightarrow 3 \pi$ decays.

1. Colangelo, Lanz, Leutwyler and Passemar [71, 72]

This is a modern update of the approach of Anisovich and Leutwyler [64], with final results still pending. Some of the crucial features are:

- The Bern $\pi \pi$ phase shifts $[73,74]$ are used with effectively six subtraction constants, which are constrained by data in the charged decay channel. 
- Electromagnetic effects are fully taken into account to NLO [62].

- To pin down the absolute magnitude, the dispersive amplitude is matched to the chiral NLO amplitude for small values of $s, t, u$. The amplitude is compatible with the Adler zero.

2. Schneider, Kubis and Ditsche [75]

These authors use a nonrelativistic effective field theory to two-loop accuracy that

- is well suited to study the dynamics of the final-state interaction and includes all isospinviolating corrections.

- It yields relations between charged and neutral Dalitz plots.

- The rescattering effects lead to sizable corrections for the Dalitz plot parameters. The approach offers an explanation why NNLO CHPT misses an important contribution to the neutral Dalitz parameter $\alpha$ defined in Eq. (6.2).

3. Kampf, Knecht, Novotny and Zdrahal [76]

This approach uses an analytic two-loop representation with the following main features.

- The amplitudes contain six parameters that are fitted to the Dalitz plot distribution of the charged decay mode.

- With these constants determined, the authors predict the parameter $\alpha$ for the neutral mode.

- They match their dispersive amplitude to the absorptive part of the NNLO chiral amplitude in a region where the differences between NLO and NNLO amplitudes are small. In fact, this is so far the only attempt to match to CHPT at the NNLO level.

The result has been criticized by the Bern group [71, 72] because the fitted amplitude is quite far away from the Adler zero.

4. Guo et al. (JPAC) [77]

This is the most recent dispersive analysis.

- The dispersive amplitude uses the Madrid/Cracow $\pi \pi$ phase shifts [78] with only three subtraction constants.

- They fit to the experimental Dalitz plot for the charged mode from the WASA/COSY Collaboration [79] and then predict the neutral Dalitz parameter $\alpha$.

- The absolute scale is fixed by matching to NLO CHPT near the Adler zero to extract a value for $Q$.

Three high-precision experiments have recently measured the Dalitz plot parameters, with the greatest accuracy provided by the new KLOE results presented at this Workshop [81]. In Table 8 I confront the experimental results with theoretical predictions, as far as available. The experimental results for the Dalitz plot parameters $a, b, d$ in the charged channel are consistent with 
each other and can therefore be averaged. The averages are clearly dominated by the KLOE results. Comparing with theoretical predictions, the least one can say is that the data present challenges for most of the theoretical approaches. In particular, NNLO CHPT has serious problems with the parameters $b$ and $\alpha$. It remains to be seen whether the new set of LECs BE14 can improve the situation.

\begin{tabular}{|c||c|c|c||c|}
\hline & $-a$ & $b$ & $d$ & $\alpha$ \\
\hline WASA/COSY 2014 [79] & $1.144(18)$ & $0.219(19)(47)$ & $0.086(18)(15)$ & \\
BESIII 2015 [80] & $1.128(15)(8)$ & $0.153(17)(4)$ & $0.085(16)(9)$ & $-0.055(14)(4)$ \\
KLOE 2015 [81] & $1.095(3)\left(_{-2}^{+3}\right)$ & $0.145(3)(5)$ & $0.081(3)\left({ }_{-5}^{+6}\right)$ & \\
my averages & $1.099(4)$ & $0.147(6)$ & $0.082(6)$ & \\
PDG 2014 [54] & & & & $-0.0315(15)$ \\
\hline NNLO CHPT [67] & $1.271(75)$ & $0.394(102)$ & $0.055(57)$ & $0.013(32)$ \\
NREFT [75] & $1.213(14)$ & $0.308(23)$ & $0.050(3)$ & $-0.025(5)$ \\
KKNZ [76] & & & & $-0.044(4)$ \\
JPAC [77] & $1.116(32)$ & $0.188(12)$ & $0.063(4)$ & $-0.022(4)$ \\
\hline
\end{tabular}

Table 8: Experimental data from three recent high-precision experiments on the Dalitz plot parameters (6.2) (charged and neutral modes). In the lower half of the table, the available theoretical results are shown for comparison.

Finally, one can extract values for the quark mass ratios from the experimental rates. In Table 9 I collect the values for the ratios $R$ and $Q$ (6.1) together with $N_{f}=2+1$ lattice averages [13]. The general tendency can be summarized in the following way. Since the Prague/Marseille group [76] matches to NNLO CHPT, their values for $R, Q$ are bigger than those from Refs. [71, 77] where the matching was performed at the NLO level. The lattice values [13] are in between.

\section{Conclusions}

Chiral perturbation theory for light mesons is still strong and healthy after more than 30 years. The main challenge for CHPT to understand the physics of the Standard Model in the confinement

\begin{tabular}{|c|c|c|c|c||c|}
\hline & NNLO CHPT [67, 82] & $\begin{array}{c}\text { CLLP [71] } \\
\text { prel. }\end{array}$ & KKNZ [76, 83] & JPAC [77] & $\begin{array}{c}\text { FLAG 2014 [13] } \\
\left(N_{f}=2+1\right)\end{array}$ \\
\hline$R$ & 40.9 & {$[31.9]^{*}$} & $37.4(2.2)$ & {$[32.2]^{*}$} & $35.8(1.9)(1.8)$ \\
$Q$ & {$[24.1]^{*}$} & $21.3(6)$ & {$[23.1]^{*}$} & $21.4(4)$ & $22.6(7)(6)$ \\
\hline
\end{tabular}

Table 9: Theoretical predictions for the quark charge ratios $R, Q$. The values without brackets are taken directly from the publications. The values in brackets with an asterisk (without errors) were calculated using the relation between $R$ and $Q$ in Eq. (6.1) and the FLAG value [13] for $m_{s} / m_{u d}$. 
regime has been met successfully. This applies especially to chiral $S U(2)$ where the longtime puzzle of the charged pion polarizabilities has now been resolved, with an impressive agreement between CHPT and experiment.

With chiral $S U(3)$, there is still ample room for improvements. The abundance of low-energy constants at NNLO is one major obstacle on the way to precision physics. A new global fit for the LECs in the strong sector is a promising step towards improving the situation. With the new set of LECs, NNLO CHPT and the most recent lattice results for the $K_{\ell 3}$ vector form factor at $t=0$ are now in excellent agreement, leading to a precise value of $V_{u s}$.

The second main construction site for chiral $S U(3)$ is the slow convergence of the chiral series in some cases. The problem has been attacked by several groups with dispersive methods, both for $K_{\ell 4}$ decays and especially for $\eta \rightarrow 3 \pi$ where CHPT seems unable to account for the measured Dalitz plot distributions even at NNLO. Although the dispersive approaches depend heavily on experimental input for pinning down the subtraction constants, they can incorporate final-state interactions more effectively than CHPT. In any case, the new very precise data for the $\eta \rightarrow 3 \pi$ Dalitz plot parameters constitute a challenge for all approaches.

The lack of evidence for new physics in the low-energy regime should not be held against CHPT. After all, CHPT is in good company with LHC physics in this respect.

\section{Acknowledgments}

I thank Hans Bijnens for allowing me to present our unpublished result for the $K_{\ell 3}$ form factor at $t=0$ and Simona Giovanella for sending me the new KLOE data for the Dalitz plot parameters prior to the meeting. Last but not least, my thanks are due to Laura Marcucci and Michele Viviani for a very inspiring and enjoyable Workshop. 


\section{References}

[1] S. Weinberg, Physica A 96 (1979) 327.

[2] J. Gasser and H. Leutwyler, Annals Phys. 158 (1984) 142.

[3] J. Gasser and H. Leutwyler, Nucl. Phys. B 250 (1985) 465.

[4] J. Bijnens and G. Ecker, Ann. Rev. Nucl. Part. Sci. 64 (2014) 149 [arXiv:1405.6488 [hep-ph]].

[5] J. Bijnens, Prog. Part. Nucl. Phys. 58 (2007) 521 [hep-ph/0604043].

[6] J. Bijnens, Eur. Phys. J. C 75 (2015) 1, 27 [arXiv:1412.0887 [hep-ph]].

[7] J. Bijnens and I. Jemos, Nucl. Phys. B 854 (2012) 631 [arXiv:1103.5945 [hep-ph]].

[8] J. Bijnens, G. Colangelo and G. Ecker, JHEP 9902 (1999) 020 [hep-ph/9902437].

[9] J. Gasser, C. Haefeli, M. A. Ivanov and M. Schmid, Phys. Lett. B 652 (2007) 21 [arXiv:0706.0955 [hep-ph]].

[10] S. Z. Jiang, Z. L. Wei, Q. S. Chen and Q. Wang, Phys. Rev. D 92 (2015) 025014 [arXiv:1502.05087 [hep-ph]].

[11] S.-Z. Jiang, Y. Zhang, C. Li and Q. Wang, Phys. Rev. D 81 (2010) 014001 [arXiv:0907.5229 [hep-ph]].

[12] G. Ecker, P. Masjuan and H. Neufeld, Eur. Phys. J. C 74 (2014) 2748 [arXiv:1310.8452 [hep-ph]].

[13] S. Aoki et al., Eur. Phys. J. C 74 (2014) 2890 [arXiv:1310.8555 [hep-lat]].

[14] G. Ecker, J. Gasser, A. Pich and E. de Rafael, Nucl. Phys. B 321 (1989) 311.

[15] R. Kaiser, Nucl. Phys. Proc. Suppl. 174 (2007) 97.

[16] M. Golterman, these Proceedings.

[17] H. Leutwyler and M. Roos, Z. Phys. C 25 (1984) 91.

[18] V. Cirigliano et al., JHEP 0504 (2005) 006 [hep-ph/0503108].

[19] P. A. Boyle et al. [RBC/UKQCD Collaboration], Phys. Rev. Lett. 100 (2008) 141601 [arXiv:0710.5136 [hep-lat]].

[20] G. Colangelo et al., Eur. Phys. J. C 71 (2011) 1695 [arXiv:1011.4408 [hep-lat]].

[21] A. Bazavov et al., Phys. Rev. Lett. 112 (2014) 112001 [arXiv:1312.1228 [hep-ph]].

[22] L. Riggio, Talk presented at 32nd Int. Symposium on Lattice Field Theory, Columbia Univ., New York, 2014.

[23] P. A. Boyle et al. [RBC/UKQCD Collaboration], JHEP 1506 (2015) 164 [arXiv:1504.01692 [hep-lat]].

[24] J. Bijnens and G. Ecker, unpublished (2015).

[25] J. Bijnens and P. Talavera, Nucl. Phys. B 669 (2003) 341 [hep-ph/0303103].

[26] A. Lusiani, "Determination of $\left|V_{u s}\right|$ from the tau lepton branching fractions", arXiv:1411.4526 [hep-ex].

[27] M. Moulson, "Experimental determination of $V_{u s}$ from kaon decays", arXiv:1411.5252 [hep-ex].

[28] J. C. Hardy and I. S. Towner, Phys. Rev. C 91 (2015) 2, 025501 [arXiv:1411.5987 [nucl-ex]]. 
[29] M. Antonelli, V. Cirigliano, A. Lusiani and E. Passemar, JHEP 1310 (2013) 070 [arXiv:1304.8134 [hep-ph]].

[30] J. Bijnens and F. Cornet, Nucl. Phys. B 296 (1988) 557.

[31] U. Bürgi, Phys. Lett. B 377 (1996) 147 [hep-ph/9602421].

[32] J. Gasser, M. A. Ivanov and M. E. Sainio, Nucl. Phys. B 745 (2006) 84 [hep-ph/0602234].

[33] J. Friedrich, these Proceedings.

[34] J. Ahrens et al. [MAMI A2 Collaboration], Eur. Phys. J. A 23 (2005) 113 [nucl-ex/0407011].

[35] L. V. Fil'kov and V. L. Kashevarov, Phys. Rev. C 73 (2006) 035210 [nucl-th/0512047].

[36] C. Adolph et al. [COMPASS Collaboration], Phys. Rev. Lett. 114 (2015) 062002 [arXiv:1405.6377 [hep-ex]].

[37] B. Pasquini, D. Drechsel and S. Scherer, Phys. Rev. C 77 (2008) 065211 [arXiv:0805.0213 [hep-ph]].

[38] J. R. Batley et al. [NA48-2 Collaboration], Eur. Phys. J. C 70 (2010) 635.

[39] G. Colangelo, J. Gasser and A. Rusetsky, Eur. Phys. J. C 59 (2009) 777 [arXiv:0811.0775 [hep-ph]].

[40] G. Colangelo, J. Gasser and H. Leutwyler, Phys. Lett. B 488 (2000) 261 [hep-ph/0007112].

[41] V. Cuplov and A. Nehme, "Isospin breaking in $K_{\ell 4}$ decays of the charged kaon", hep-ph/0311274.

[42] P. Stoffer, Eur. Phys. J. C 74 (2014) 2749 [arXiv:1312.2066 [hep-ph]].

[43] V. Bernard, S. Descotes-Genon and M. Knecht, Eur. Phys. J. C 73 (2013) 2478 [arXiv:1305.3843 [hep-ph]].

[44] V. Bernard, S. Descotes-Genon and M. Knecht, Eur. Phys. J. C 75 (2015) 4, 145 [arXiv:1501.07102 [hep-ph]].

[45] M. Knecht, these Proceedings.

[46] G. Amoros, J. Bijnens and P. Talavera, Nucl. Phys. B 585 (2000) 293 [Nucl. Phys. B 598 (2001) 665] [hep-ph/0003258].

[47] G. Colangelo, E. Passemar and P. Stoffer, Eur. Phys. J. C 75 (2015) 172 [arXiv:1501.05627 [hep-ph]].

[48] J. Stern, H. Sazdjian and N. H. Fuchs, Phys. Rev. D 47 (1993) 3814 [hep-ph/9301244].

[49] S. Pislak et al., Phys. Rev. D 67 (2003) 072004 [Erratum ibid. D 81 (2010) 119903] [hep-ex/0301040].

[50] J. R. Batley et al. [NA48/2 Collaboration], Phys. Lett. B 715 (2012) 105 [Addendum ibid. B 740 (2015) 364] [arXiv:1206.7065 [hep-ex]].

[51] V. Cirigliano et al., Rev. Mod. Phys. 84 (2012) 399 [arXiv:1107.6001 [hep-ph]].

[52] N. Garron, these Proceedings.

[53] Z. Bai et al. (RBC/UKQCD Collaboration), "Standard-model prediction for direct CP violation in $K \rightarrow \pi \pi$ decay", arXiv:1505.07863 [hep-lat].

[54] K. A. Olive et al. [Particle Data Group Collaboration], Chin. Phys. C 38 (2014) 090001.

[55] V. Cirigliano, A. Pich, G. Ecker and H. Neufeld, Phys. Rev. Lett. 91 (2003) 162001 [hep-ph/0307030]. 
[56] V. Cirigliano, G. Ecker, H. Neufeld and A. Pich, Eur. Phys. J. C 33 (2004) 369 [hep-ph/0310351].

[57] R. J. Crewther and L. C. Tunstall, Phys. Rev. D 91 (2015) 3, 034016 [arXiv:1312.3319 [hep-ph]].

[58] L. C. Tunstall, these Proceedings.

[59] D. G. Sutherland, Phys. Lett. 23 (1966) 384.

[60] J. S. Bell and D. G. Sutherland, Nucl. Phys. B 4 (1968) 315.

[61] R. Baur, J. Kambor and D. Wyler, Nucl. Phys. B 460 (1996) 127 [hep-ph/9510396].

[62] C. Ditsche, B. Kubis and U. G. Meißner, Eur. Phys. J. C 60 (2009) 83 [arXiv:0812.0344 [hep-ph]].

[63] J. Kambor, C. Wiesendanger and D. Wyler, Nucl. Phys. B 465 (1996) 215 [hep-ph/9509374].

[64] A. V. Anisovich and H. Leutwyler, Phys. Lett. B 375 (1996) 335 [hep-ph/9601237].

[65] H. Osborn and D. J. Wallace, Nucl. Phys. B 20 (1970) 23.

[66] J. Gasser and H. Leutwyler, Nucl. Phys. B 250 (1985) 539.

[67] J. Bijnens and K. Ghorbani, JHEP 0711 (2007) 030 [arXiv:0709.0230 [hep-ph]].

[68] R. F. Dashen, Phys. Rev. 183 (1969) 1245.

[69] S. L. Adler, Phys. Rev. 137 (1965) B1022.

[70] B. Moussallam, these Proceedings.

[71] G. Colangelo, S. Lanz, H. Leutwyler and E. Passemar, PoS EPS-HEP2011 (2011) 304 and work in progress.

[72] H. Leutwyler, Mod. Phys. Lett. A 28 (2013) 1360014 [arXiv:1305.6839 [hep-ph]].

[73] B. Ananthanarayan, G. Colangelo, J. Gasser and H. Leutwyler, Phys. Rept. 353 (2001) 207 [hep-ph/0005297].

[74] G. Colangelo, J. Gasser and H. Leutwyler, Nucl. Phys. B 603 (2001) 125 [hep-ph/0103088].

[75] S. P. Schneider, B. Kubis and C. Ditsche, JHEP 1102 (2011) 028 [arXiv:1010.3946 [hep-ph]].

[76] K. Kampf, M. Knecht, J. Novotny and M. Zdrahal, Phys. Rev. D 84 (2011) 114015 [arXiv:1103.0982 [hep-ph]] and work in progress.

[77] P. Guo et al. [JPAC] Phys. Rev. D 92 (2015) 5, 054016 [arXiv:1505.01715 [hep-ph]].

[78] R. Garcia-Martin et al., Phys. Rev. D 83 (2011) 074004 [arXiv:1102.2183 [hep-ph]].

[79] P. Adlarson et al. [WASA-at-COSY Collaboration], Phys. Rev. C 90 (2014) 4, 045207 [arXiv:1406.2505 [hep-ex]].

[80] M. Ablikim et al. [BESIII Collaboration], Phys. Rev. D 92 (2015) 1, 012014 [arXiv:1506.05360 [hep-ex]].

[81] S. Giovanella (KLOE), these Proceedings.

[82] J. Bijnens, numerical update of Ref. [67] (private communication).

[83] M. Knecht, numerical update of Ref. [76] (private communication). 